\title{
A STUDY OF THE CLOSED-LOOP DIRECTIONAL STABILITY OF VARIOUS COMMERCIAL VEHICLE CONFIGURATIONS
}

\author{
Charles C. MacAdam and Paul S. Fancher
}

The University of Michigan, Transportation Research Institute, Ann Arbor, Michigan, 48109, USA.

SUMMARY

Computer analysis of the closed-loop directional stability of four common commercial vehicle configurations was performed using 1) a disturbance input technique to study low lateral acceleration driving conditions, and 2) a lane-change maneuver for studying system response at elevated lateral acceleration conditions. The results of the disturbance input calculations indicated, that under low lateral acceleration conditions, drivers of different commercial vehicle configurations should be capable of providing adequate control compensation to achieve more or less equal stability margins for most systems. Evaluation of directional stability, based upon the transient lane-change maneuver, indicated systematic reduction in system damping for all vehicles with increased levels of lateral acceleration. The 5-axle tractor-semitrailer system exhibited the greatest level of directional damping and rollover immunity during the lane-change maneuver. The truck full-trailer was the least damped system while also producing rollover occurrences for the least lateral lane-change displacement. Use of the disturbance input test, as employed here, is not applicable for examining the total system stability of vehicle configurations employing single pintle-hook (or "A-do1ly") hitching arrangements and which also exhibit rearward amplification tendancies. Train units rearward of such hitching mechanisms lie outside the closed-loop control structure of the driver. Recommendations for improving the closed-loop control structure of these systems are offered.

\section{INTRODUCTION}

This paper examines, through computer simulation, the degree to which different types of commercial vehicle configurations can influence and promote different levels of closed-loop directional stability for representative driver-vehicle systems. Several types of vehicles are studied including a straight truck, tractor-semitrailer, truck-full trailer, and doubles combination. A comprehensive vehicle dynamics mode1 $[1,2]$ is used to simulate the dynamic response of each of the 
respective vehicle types. A well documented driver model [3] is used to represent the closed-loop steering properties of the simulated driver. Nominal driver characteristics, determined from previous driver-vehicle research and existing experimental measurements $[3,4,5]$, are assumed within this study. Although related studies conducted for passenger car-driver systems have been reported in the technical literature, the results presented in this paper focus on the predicted interaction between heavy truck dynamics and driver steering control. Furthermore, several analysis techniques used in this paper are viewed as useful tools having potential application to the more general vehicle dynamics field.

Experimental studies of man-machine systems routinely observe a certain degree of compensatory or modified human operator behavior in response to variations in the dynamics of the system being controlled $[5,6]$. In some cases, this observed compensatory behavior on the part of the human operator is sufficient to cause the dynamics of the total man-machine (closed-1oop) system to remain nearly invariant despite significant changes to the controlled system dynamics. In other cases, only partial compensation by human operators is achievable, thereby resulting in a net change of total man-machine system performance and stability. This same basic phenomena, examined within the context of commercial vehicle dynamical systems, is the focus of this paper. Since the driver model employed within this study contains compensatory/adaptive characteristics represented by an internal model of the particular vehicle being controlled, behavior patterns similar to those observed in many experimental studies can be represented. Results of the study include predictions of the extent to which closed-loop stability margins can vary as changes in vehicle configurations occur. Additional results are presented to illustrate the influence of lateral acceleration and maneuver severity on the degree of closed-loop system stability. Two types of closed-loop maneuvers are used to investigate the issues discussed above: 1) low and moderate lateral acceleration maneuvers performed in the presence of a random-like steering disturbance, and 2) lane-change maneuvers performed at moderate and high levels of lateral acceleration. The random disturbance maneuvers are used primarily to characterize locally linearized system characteristics and stability margins of each of the drivervehicle systems at low and moderate lateral acceleration operating conditions. Results of the random disturbance maneuvers are presented using frequency domain methods commonly employed in control system analyses $[7,8]$. The lane change maneuvers are used to examine the performance of these same systems within the time domain and primarily at elevated levels of lateral acceleration. Sensitivities of closed-1oop system performance and stability to increased levels of non-linear vehicle behavior are also studied by means of the lane change maneuver. 


\section{DISTURBANCE INPUT METHODOLOGY}

Figures 1 through 3 help to illustrate the basic concept of the disturbance maneuver as used here. Figure 1 shows the two main elements comprising a driver/vehicle system and the addition of an input disturbance, dd, to the driver steering control.

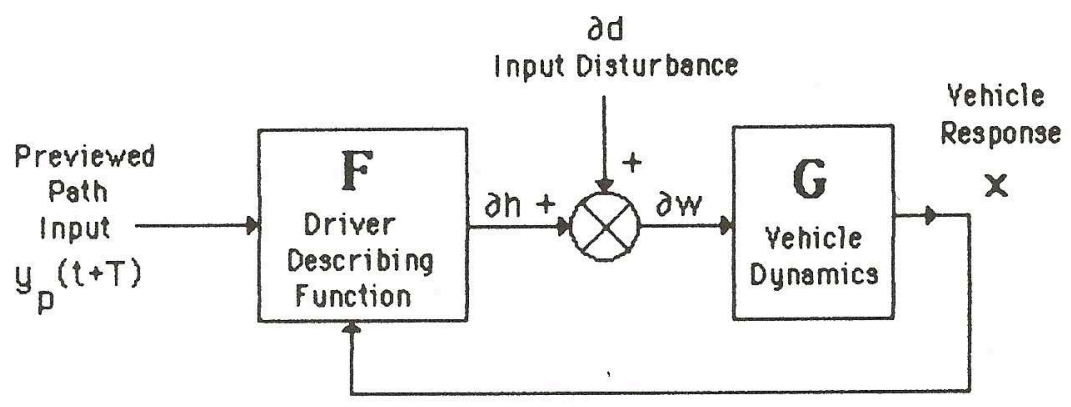

Fig. 1. Driver/Vehicle System with an Applied Steering Disturbance.

The input to the vehicle, $d w$, is the sum of the driver steering output, ah, and the known random-like disturbance, ad. The driver control task is to follow a previewed path while simultaneously compensating for the small steering disturbance. The vehicle response given by the state vector, $x$, and the previewed path input, $y(t+T)$, are both processed by the driver within the driver describing function block, $F$, to produce a steering control. The parameter, $T$, represents the preview interval over which the driver is observing the desired path.

Figure 2 is a rearrangement of Figure 1 and an attempt to separate the driver describing function into two more specific operational blocks usually associated with the driving process. (The driver model [3] employed in this study is very similar in structure to that shown in Figure 2.) Block $P$ represents the ability of a driver to predict future vehicle position based upon an understanding of the vehicle dynamics and the current vehicle response. Block $H$ represents the basic control function and response limitation of the driver. Block $H$ operates on an assumed previewed path error signal, $e(t+T)$, which is formed as the difference between the previewed path, $y_{(}(t+T)$, and the future vehicle position predicted by the driver, $y(t+T)$.

Under applicable conditions, knowledge or measurement of ad and $d_{w}$ (or dh) permit the estimation of the combined (open-loop) driver/vehicle transfer function, $(F * G)$, or, $(H * G * P)$. The Fourier transform of the quantity, -ah / $\mathrm{dw}$, or equivalently, (ad / $\partial w-1)$, provides the desired driver/vehicle transfer function. Conventional control system methods may then be applied for analyzing the stability of the closed-loop driver/vehicle system.

Under conditions of straight-line driving in the presence of an applied disturbance, Figure 3 may be derived from Figure 2 and 

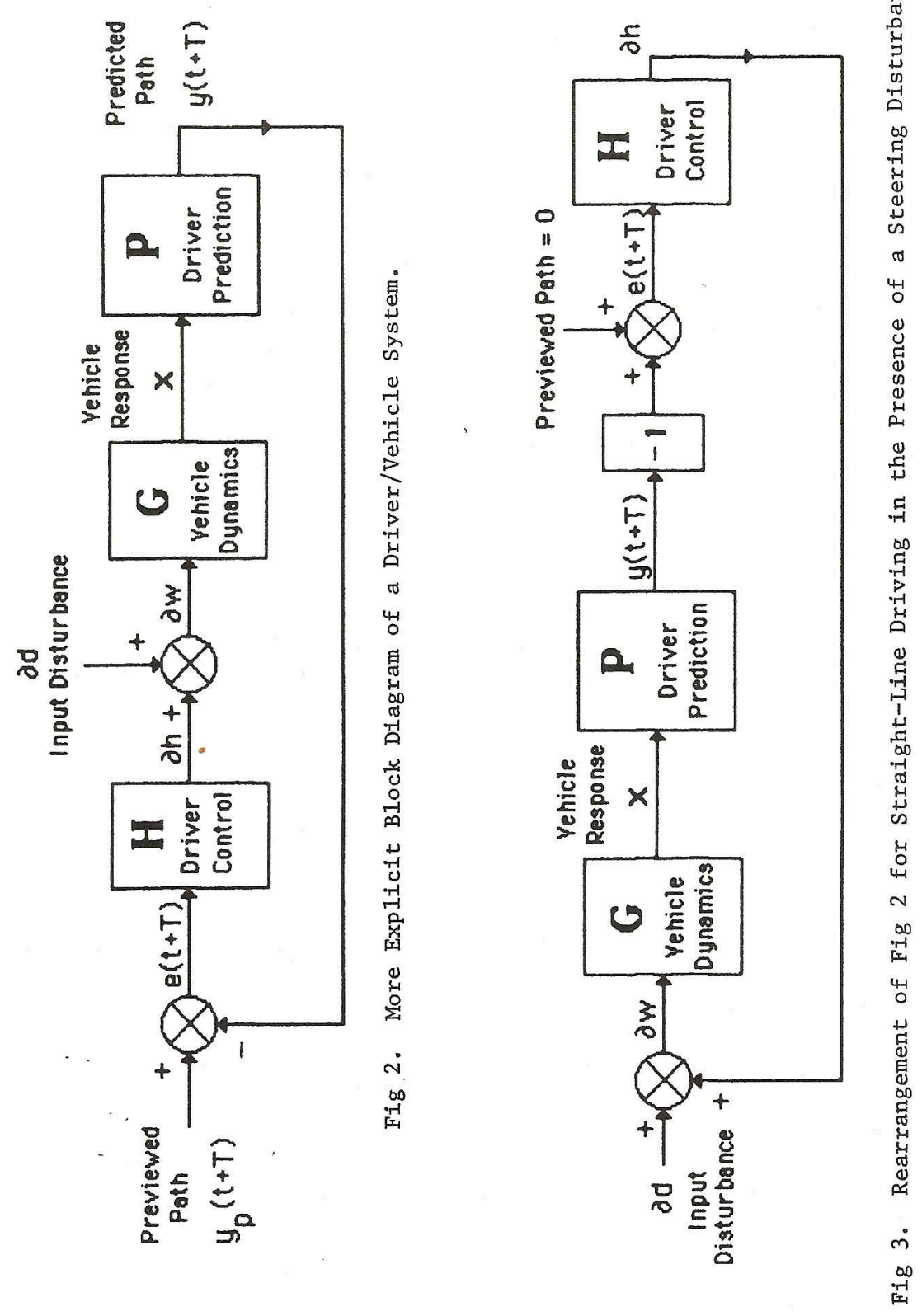
interpreted directly as the driver steering action in response to the disturbance input. An ideal driver response would exactly cancel the disturbance input so that the vehicle steer input, dw, is zero. However, because of driver transport lags and vehicle dynamic characteristics, the actual driver response is not perfect. Accordingly, the closed-loop driver/vehicle system will exhibit different levels of stability depending upon driver and/or vehicle properties.

Figure 4 is an example open-loop frequency response calculation, $H^{*} G^{*} P$, for a simulated driver/truck system. The top figure represents the open-loop gain of the driver-vehicle system; the bottom portion is the corresponding phase. These data were obtained by calculating the Fourier transform of the quantity ( $\partial \mathrm{d} /$ $\partial w$ - 1) recorded from a disturbance input run. Comparison of these data with comparable experimental measurements of driver-passsenger car systems [5] show nearly identical characteristics being exhibited by the computer model. As seen, "crossover model" behavior typically observed in most man-machine experimental data is also displayed by the simulated driver/vehicle system seen here.

The results seen in Figure 4 may also be plotted on a Nichols chart in order to evaluate the stability of the closed-1oop system $[7,8]$. Figure 5 shows these same results plotted on a Nichols chart. The ordinate and abscissa axes represent the gain and phase of the open-1oop system seen in Figure 4. The interior M-contours and p-contours reveal the corresponding gain and phase of the closedloop system. The basic measure of closed-loop stability is provided on a Nichols chart by the point of nearest approach of the frequency response locus to the ( $0 \mathrm{db},-180$ degree) point (largest M-contour value). Examination of Figure 5 shows that the peak closed-1oop system resonance occurs at about $2.5 \mathrm{rad} / \mathrm{sec}$ with a value of about 6 $\mathrm{db}$ on the M-contour. The entire closed-loop system response could of course be plotted as a function of frequency in this same manner.

To illustrate, Figure 6 shows the corresponding closed-1oop frequency response gain and phase data derived from the interior contours of the Nichols chart. The example plot in Figure 6 shows the peak resonance or amplification of the disturbance input by the driver occurring at 2.5 radians/second. The corresponding closedloop phase falls off with increasing 1 ag as frequency content increases. (Recall that the ideal response is one having a 0 db gain and -180 degrees of phase shift indicating perfect cancellation of the disturbance input by the driver). In general, increased driver preview times or decreased driver transport lags wil1 attenuate the observed resonance and tend to increase the stability of the closedloop driver/vehicle system. Likewise, shorter driver preview times or greater driver lags will diminish the degree of stability. Vehicle parameter variations may also be studied in this manner to evaluate their influence. The following section shows results of applying this technique to four different driver/vehicle configurations for examining relative levels of closed-loop stability under conditions of low lateral acceleration. 

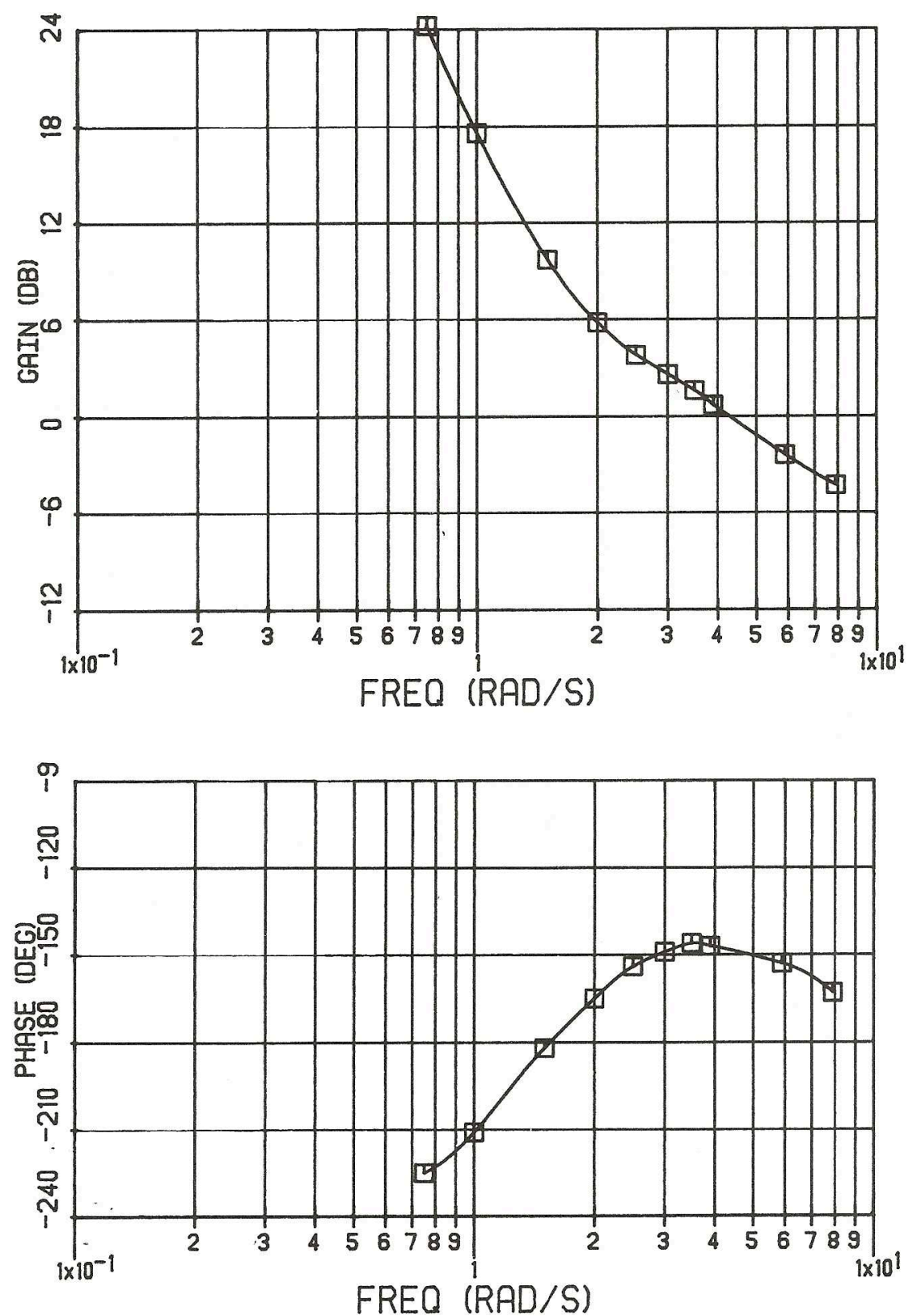

Fig. 4. Example Calculation of a Driver/Truck (open-1oop) Frequency Response, $H^{*} G * P$. 


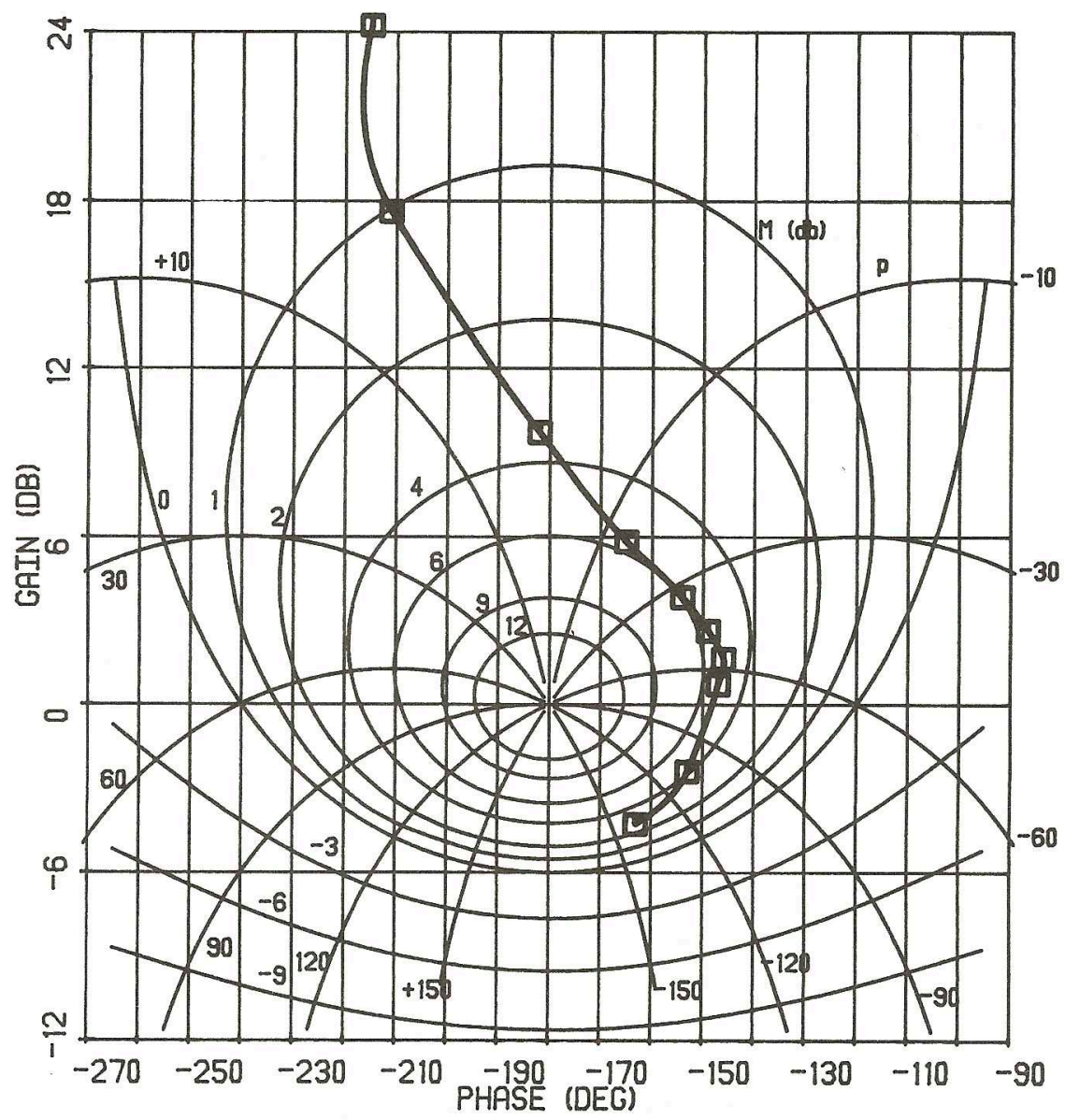

Fig. 5. Nichols Chart Corresponding to Fig 4 Result.

\section{DISTURBANCE INPUT RESULTS}

Disturbance input runs were performed for four different commercial vehicle configurations. The basic descriptions of each vehicle are shown in Table 1 and are considered representative of certain fullyloaded vehicles within each general vehicle class. The results presented in this paper are, of course, specific to the vehicles examined here and are not intended to generically represent all "Doubles" or all "Straight-Trucks." Driver model adaptation to differences in basic vehicle dynamics were permitted to occur in these calculations, but driver preview and delay times were held fixed at $1.5 \mathrm{sec}$ and $0.25 \mathrm{sec}$, respectively. 


\section{3-Axle \\ Straight \\ Truck}

Closed-Loop Frequency Response

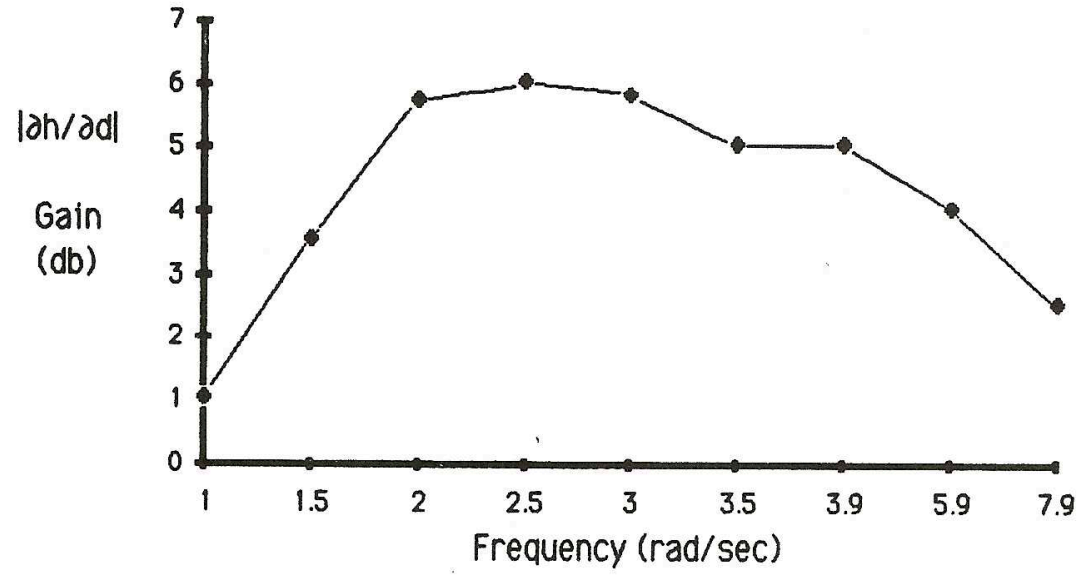

Closed-Loop Frequency Response

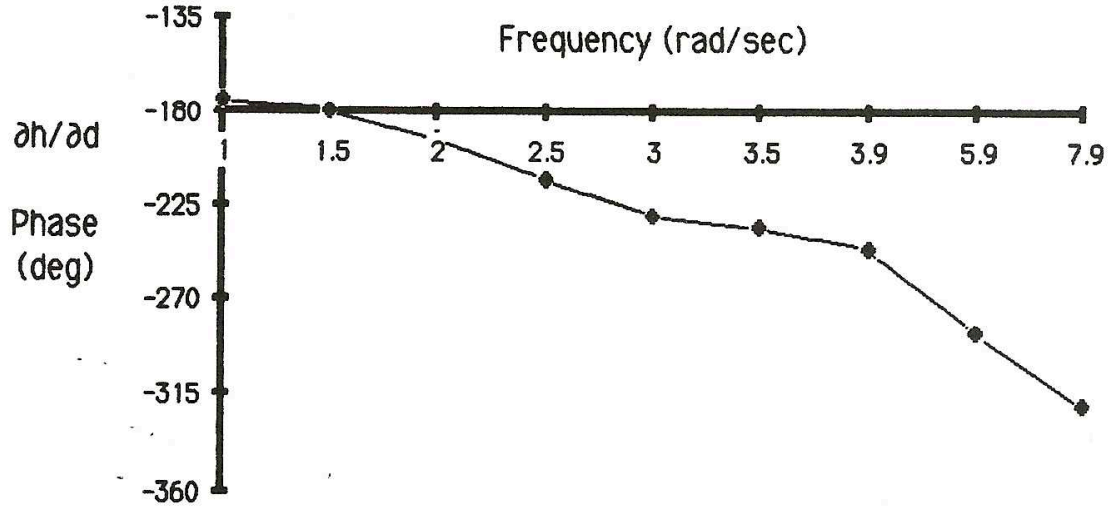

Fig. 6. Closed-Loop Frequency Response Derived from the Inner Contours of Fig 5 . 
Table 1. Vehicle Descriptions

Vehicle Type Axle Loads (kN) Wheelbase (m) C.G. Height (m)

Straight-Truck $53 / 76 / 76$

6.05

1.93

Tractor-Semitrailer

$53 / 76 / 76 / 76 / 76$

$3.68 / 10.9$

$1.12 / 2.00$

Doub1e

$44 / 78 / 78 / 78 / 7$

Truck Ful1-Trailer

$47 / 70 / 70 / 85 / 85$

$6.0 / 5.6$

$1.8 / 2.0$

Disturbance input runs identical to those described in Section 2 were conducted for each of these baseline vehicle configurations to compare the degree to which they differed. Results were compared in terms of the peak closed-1oop resonances displayed by each driver/vehicle configuration. The same performance measures were also compared for identical disturbance input tests conducted during circular turning conditions of $0.25 \mathrm{~g}^{\circ} \mathrm{s}$. The purpose of the circular turning disturbance test was to evaluate the degree to which modest lateral acceleration levels affected the stability of the closed-1oop systems. A typical result for the $0 \mathrm{~g}$ (straight-line) and $0.25 \mathrm{~g}$ turning test for the Double is shown in Figure 7. The peak resonance value of $11 \mathrm{db}$ at $2 \mathrm{rad} / \mathrm{sec}$ for the $0.25 \mathrm{~g}$ turning condition, versus the $6 \mathrm{db}$ value at $2.5 \mathrm{rad} / \mathrm{sec}$ for the $0 \mathrm{~g}$ condition, suggests reduced stability of the same closed-loop system at the elevated lateral acceleration condition. Because the driver model used here initializes its control characteristics to the linear vehicle dynamics of the lead unit under static load ( or $0 \mathrm{~g}$ ) conditions, vehicles or maneuvers that cause significant departure from the internal representation of the vehicle dynamics assumed by the driver model will exhibit variations in closed-loop stability which largely reflect non-adaptive control characteristics of drivers. Since adaptive compensation by the driver model is not occurring in the $0.25 \mathrm{~g}$ calculation, Figure 7 represents the change in closed-1oop stability deriving primarily from changes to the vehicle directional dynamics induced by lateral acceleration.

Figure 8 contains a summary of peak closed-1oop resonances for each of the baseline vehicles obtained from corresponding plots identical to the one shown in Figure 7. The comparisons in Figure 8 show that for straight-line driving conditions all of the driver/vehicle combinations display approximately the same level of stability with peak closed-loop resonance values in the vicinity of 6 $\mathrm{db}$. This result is largely due to the driver model's ability to properly identify the basic directional dynamics of each vehicle under low lateral acceleration conditions and thereby provide compensation for vehicle-to-vehicle differences in directional dynamics. However, the circular turning disturbance results show that the Straight-Truck and the Double exhibit significantly lower levels of stability in response to elevated levels of lateral acceleration as noted by the increased resonance values of $8.5 \mathrm{db}$ and $11 \mathrm{db}$ respectively. The noted sensitivities would imply that the 


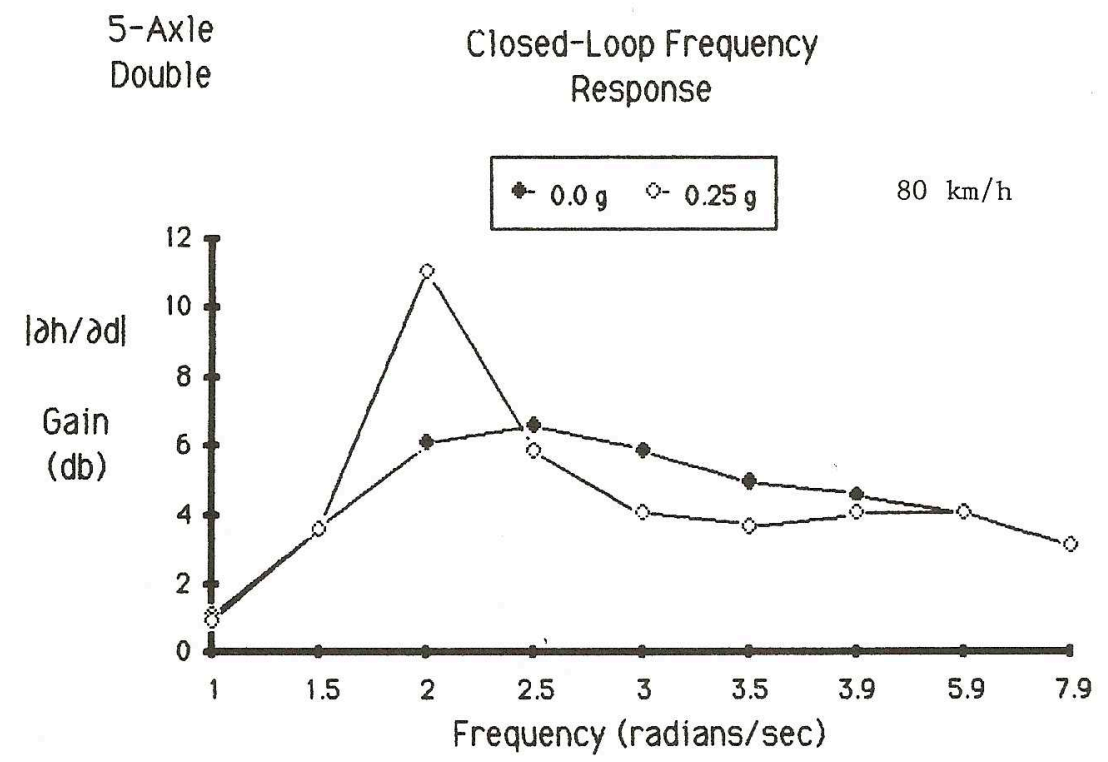

Fig. 7. Example Closed-Loop Frequency Response for the Double; Straight-Line Running vs. $0.25 \mathrm{~g}$ Cornering.

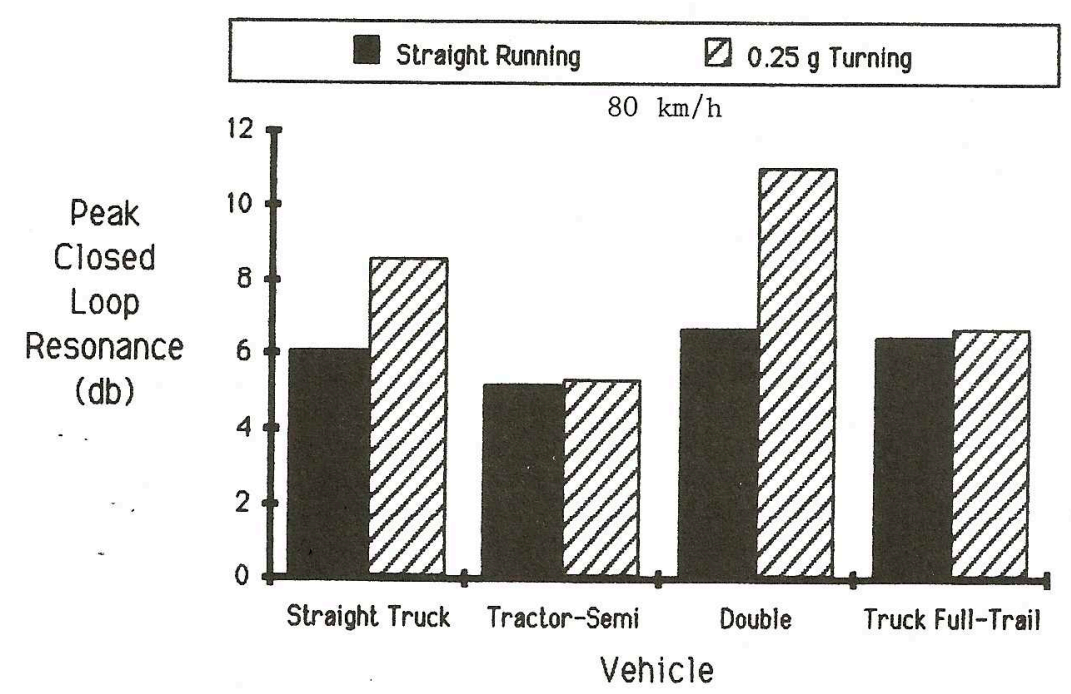

Fig. 8. Vehicle Comparison of Peak Closed-Loop Resonances. 
Straight-Truck and the Double demand greater control compensation on the part of drivers durfing increased lateral acceleration conditions to maintain a stability margin comparable to that observed during straight-line driving conditions.

\section{LANE-CHANGE RESULTS}

In addition to the disturbance-input runs, a series of $80 \mathrm{~km} / \mathrm{h}$ lanechange maneuvers were also performed to evaluate the same driver/vehicle systems during moderate and high lateral acceleration obstacle-avoj.dance conditions. The basic methodology was to perform a sequence of lane-change maneuvers of increasing lateral

displacement, keeping the longltudinal travel distance fixed at 30.5 $\mathrm{m}$. By increasing the lateral displacement requirement, increasingly higher lateral accelerations were achieved with an eventual limit determined by a rollover occurrence. Measures of system damping and path overshoot were also recorded as a function of required lateral displacement to illustrate and evaluate degradation of closed-1oop system performance under higher lateral acceleration conditions.

Figure 9 shows a typical sequence of three lateral displacement and lateral acceleration time histories for the Straight-Truck obtained by performing lane-change maneuvers of $2.4 \mathrm{~m}, 3.6 \mathrm{~m}$, and 4.8 $\mathrm{m}$, respectively. A $5.2 \mathrm{~m}$ lane-change produced rollover of the vehicle and thereby defined the upper limit for this maneuver sequence. Similar calculations were performed for the remaining three driver/vehicle combinations.

Figure 10 shows a particular measure of system damping, defined as the ratio of the first and third peak values of the lateral acceleration time history (Ay3 / Ay1), plotted for each vehicle and lateral lane displacement. (The last trailer of each multiple-unit vehicle is used as the reference unit for the performance measures presented in this Section.) Figure 11 presents a similar measure of system damping defined as the percent overshoot of the path of the vehicle mass center beyond its target lateral displacement. As path displacements (and corresponding maximum lateral acceleration levels) are increased, the degree of damping displayed by these two measures is seen to systematically decrease for each of the driver/vehicle combinations.

The limit condition for this maneuver is given by the maximum lateral displacement that could be achieved by the driver/vehicle combination prior to a rollover occurrence. Figure 12 summarizes this condition by plotting the minimum lane-change lateral displacement that produced a rollover instability for the vehicle during the defined closed-loop maneuver. Interestingly, the TractorSemitrailer stands out as a vehicle having far greater immunity to rollover in the lane-change maneuver than any of the three other vehicles studied. As seen in Figure 12, a lateral displacement of 9 m (accompanied by a peak 1atera1 acceleration of $0.43 \mathrm{~g}^{\prime} \mathrm{s}$ ) was required before rollover of the semitrailer could be achieved. This is in contrast to the remaining three vehicles which required lateral displacements of approximately $5 \mathrm{~m}$ for the same result. Furthermore, the Tractor-Semitrailer exhibits far greater damping, particularly 

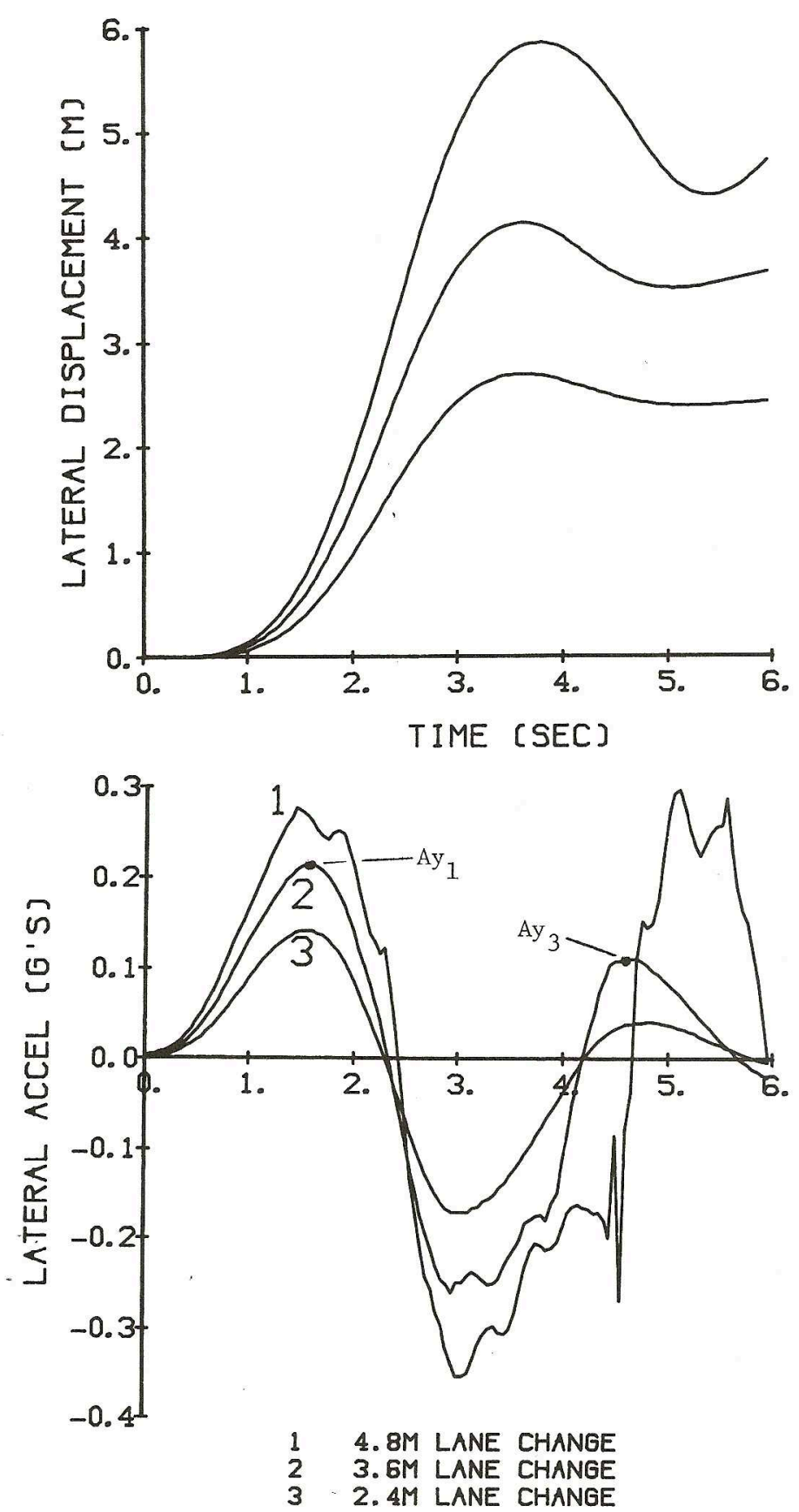

Fig. 9. Example Lane-Change Time History Results for the Straight-Truck. 
$2.4 \mathrm{~m} \square 3.6 \mathrm{~m} 4.2 \mathrm{~m} \quad 0.8 \mathrm{~m} 7.4 \mathrm{~m}$ 目 $8.6 \mathrm{~m}$

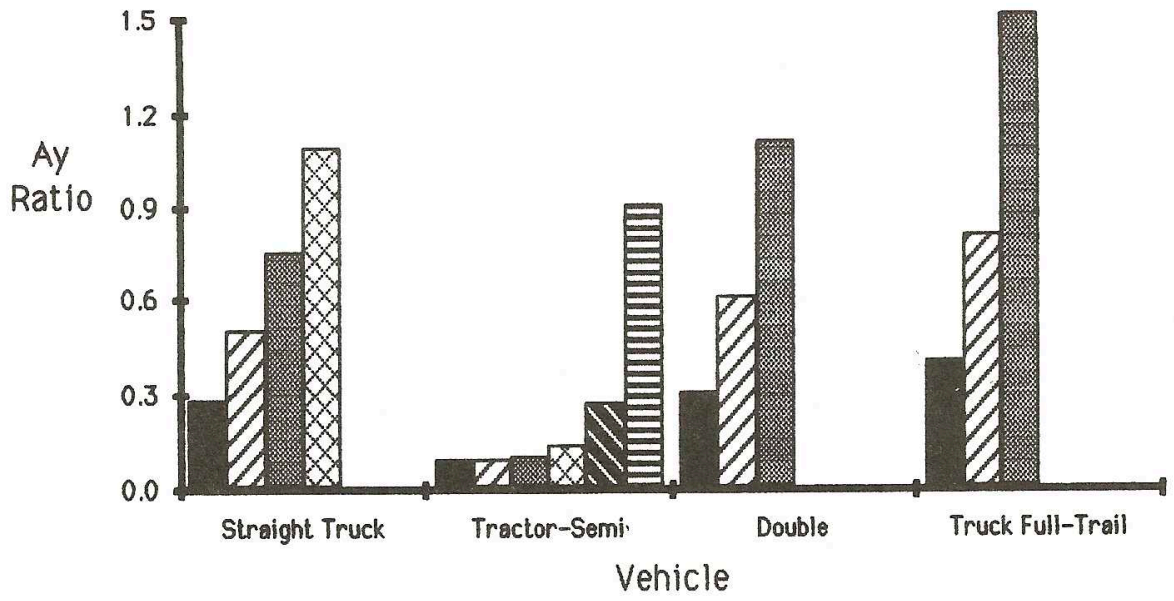

Fig. 10. $\mathrm{Ay}_{3} / \mathrm{Ay}$ I Ratio Peasure of System Damping.

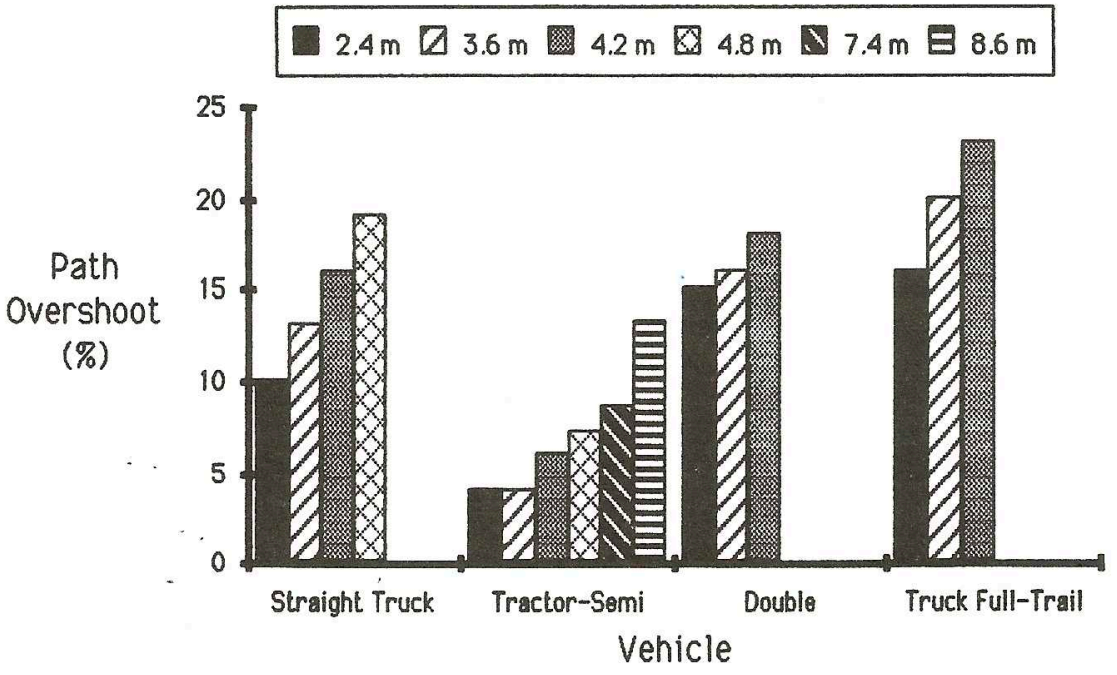

Fig. 11. Path Overshoot Measure of System Damping. 
for maneuvers that are likely to be performed within the lateral constraints of an actual highway setting.

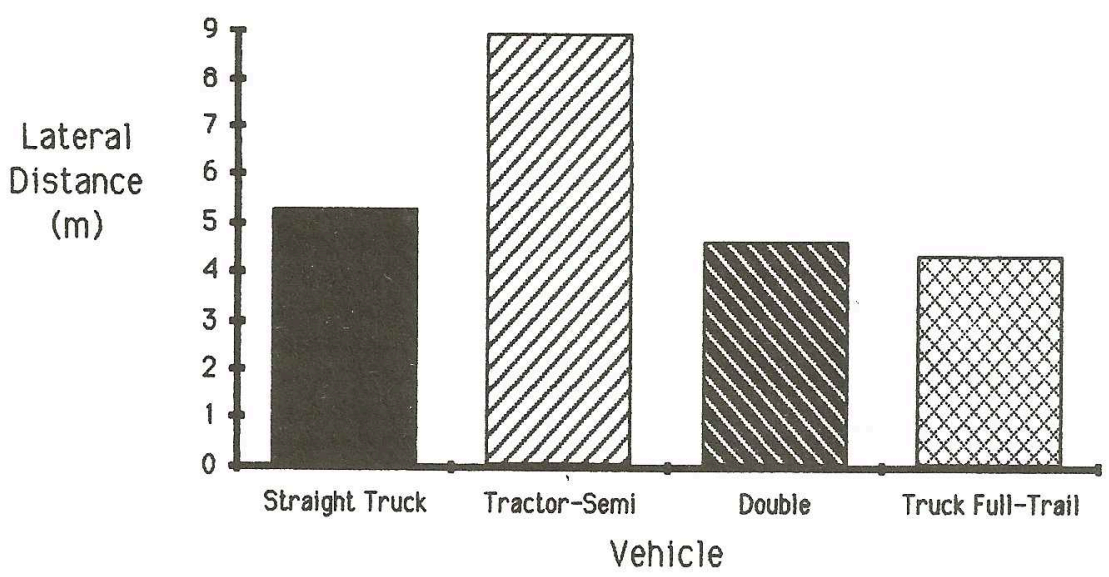

Fig. 12. Maximum Iane-Change Achieved Prior to a Rollover.

A comparison of the lane-change results and the disturbance input results reveals similar trends of reduced stability due to increased lateral acceleration levels, except for the Truck Ful1Trailer. The Truck Full-Trailer exhibits the least stability of all the systems for the lane-change maneuvers, but shows little change in stability during the disturbance input tests under increased lateral acceleration conditions. The explanation for this conflict is that the lane-change stability evaluations shown here apply to the rearmost and least stable vehicle unit, while the disturbance-input results apply to the lead unit. If, as for truck full-trailers, little dynamic coupling exists between the lead unit and its more rearward units, motion and force cues from the rear units which normally influence the lead unit (and consequently driver steering response) will not be accounted for in disturbance input tests of such vehicles. Drivers of such vehicle configurations have no true closed-loop control over the rear units. Forced dynamic responses of the full trailer are therefore determined by hitching kinematics and path inputs independently selected by closed-loop driver behavior of the lead unit. Closed-loop stability evaluations of such systems are consequently muddled because of the incomplete closed-1oop control structure introduced by the hitching mechanisms used on these vehicles:

\section{CONCLUSIONS}

The results of the disturbance input calculations indicate, that under low lateral acceleration conditions, drivers of different commercial vehicle configurations should be capable of providing adequate control compensation to achieve more or less equal stability 
margins for different closed-loop systems. This assumes that drivers employ similar control stategies and are capable of adapting to the directional dynamics of the different vehicles being controlled.

Evaluation of directional stability for the different vehicle configurations, based upon the transient lane-change maneuver, indicated systematic reduction in system damping for all vehicles at increased levels of lateral acceleration. The 5-axle TractorSemitrailer system exhibited the greatest level of directional damping and rollover immunity during the lane-change maneuver. The Truck Ful1-Trailer was the least damped system while also producing rollover occurrences for the least lateral lane-change displacement. Use of the disturbance input test, as employed here, is not applicable for examining the total sytstem stability of vehicle configuraticns employing single pintle-hook (or "A-dolly") hitching arrangements and which exhibit rearward amplification tendancies. Train units rearward of such hitching mechanisms lie outside the closed-loop control structure of the driver. As such, the dynamic response of those rearward units present no feedback control cues to drivers and therefore depend entirely upon the steering mechanism design of the hitch. For "short" trains, such as truck fulltrailers, use of a modified hitching mechanism to introduce force and/or moment feedback cues to the lead unit would improve the controllability of such systems by bringing the full-trailer within the closed-loop control structure of the driver. For "long" trains, such as doubles or triples, where lengthy time lags are present in the forward propagation of rear unit responses, the ability of drivers to employ such cues effectively would seem unlikely. A more direct approach would be to significantly improve the steering/hitching mechanisms in question so that rearward amplification tendancies are reduced to such a level that trailing units can not be amplified by units closest to and within the closedloop control structure of the driver.

\section{REFERENCES}

1. Mallikarjunarao, C., "Road Tanker Design: Its Influence on the Risk and Economic Aspects of Transporting Gasoline in Michigan," Ph.D Dissertation, The University of Michigan, December 1981.

2. Gillespie, T.D. and MacAdam, C. C., "Constant Velocity Yaw/Roll Program User's Manual," University of Michigan Transportation Research Institute, Report No. UMTRI-82-39, October 1982 .

3. MacAdam, C. C., "Application of an Optimal Preview Control for Simulation of Closed-Loop Automobile Driving," IEEE Transactions on Systems, Man, and Cybernetics, Vol. SMC-11, No. 6, June 1981, pp. 393-399.

4. McRuer, D. T. et al., "Measurement of Driver/Vehicle Multiloop Response Properties with a Single Disturbance Input," IEEE Transactions on Systems, Man, and Cybernetics, Vol. $5, \overline{\text { No. }}$ $5,1975$. 
5. Weir, D. H., DiMarco, R. J., and McRuer, D. T., "Evaluation and Correlation of Driver/Vehicle Data," Technical Report, DOTHS-5-01200.

6. Elkind, J. I., "A Survey of the Development of Models for the Human Controller," Progress in Astronautics and Aeronautics, Vo1. 13, 1964, pp. 623-643.

7. D'Azzo, J. J. and Houpis, C. H., Linear Control System Analysis and Design, Second Edition, 1981, McGraw-Hil1 Book Company, New York.

8. MacAdam, C. C., "Frequency Domain Methods for Analyzing the Closed-Loop Directional Stability and Maneuverability of Driver/Vehicle Systems," Proceedings of the International Conference on Modern Vehicle Design Analysis, London, June 1983. 


\title{
THE DYNAMICS OF VEHICLES on roads and on tracks
}

\author{
Edited by \\ Olle NORDSTRÖM \\ Proceedings of 9 th IAVSD Symposium \\ held at Linköping University, Linköping, \\ Sweden, June 24-28, 1985 \\ International Association for Vehicle System Dynamics \\ affiliated to the \\ International Union of Theoretical and Applied Mechanics
}

Supplement to

Vehicle System Dynamics, Volume 15

SWETS NORTH AMERICA INC. / BERWYN

SWETS \& ZEITLINGER B.V. / LISSE

1986 